\title{
Mapping Local Deformation Behavior in Single Cell Metal Lattice Structures
}

Holly D. Carlton ${ }^{1}$, Jonathan Lind ${ }^{1}$, Mark C. Messner ${ }^{1}$, Nickolai A. Volkoff-Shoemaker ${ }^{1}$, Harold

S. Barnard ${ }^{2}$, Nathan R. Barton ${ }^{1}$, and Mukul Kumar ${ }^{1}$

${ }^{1}$ Lawrence Livermore National Laboratory, 7000 East Avenue, L-342, Livermore, CA 94550, USA

2Lawrence Berkeley National Laboratory, 1 Cyclotron Rd, Berkeley, CA 94720, USA

\begin{abstract}
The deformation behavior of metal lattice structures is extremely complex and challenging to predict, especially since strain is not uniformly distributed throughout the structure. Understanding and predicting the failure behavior for these types of light-weighting structures is of great interest due to the excellent scaling of stiffness- and strength- to weight ratios they display. Therefore, there is a need to perform simplified experiments that probe unit cell mechanisms. This study reports on high resolution mapping of the heterogeneous structural response of single unit cells to the macro-scale loading condition. Two types of structures, known to show different stress-strain responses, were evaluated using synchrotron radiation micro-tomography while performing in-situ uniaxial compression tests to capture the localized micro-strain deformation. These structures included the octet-truss, a stretch-dominated lattice, and the rhombic-dodecahedron, a bend-dominated lattice. The tomographic analysis showed that the stretch- and benddominated lattices exhibit different failure mechanisms and that the defects built into the structure cause a heterogeneous localized deformation response. Also shown here is a change in failure mode for stretch-dominated lattices, where there appears to be a
\end{abstract}

(C) 2017. This manuscript version is made available under the Elsevier user license http://www.elsevier.com/open-access/userlicense/1.0/ 
transition from buckling to plastic yielding for samples with a relative density between 10 and $20 \%$. The experimental results were also used to inform computational studies designed to predict the mesoscale deformation behavior of lattice structures. Here an equivalent continuum model and a finite element model were used to predict both localized strain fields and mechanical behavior of lattices with different topologies.

\section{Keywords:}

Mechanical properties, Lattice structure, Synchrotron radiation computed tomography, In situ compression test

\section{Introduction}

Light-weighting metal lattice structures are being studied extensively due to their load bearing properties and low density [1-5], especially in the biomedical [6-8] and aerospace [9] industries where the tradeoff between strength and weight is very important $[10,11]$. With the advent of additive manufacturing (AM) methods, such as Selective Laser Melting $\left(\mathrm{SLM}^{\mathrm{TM}}\right)$, a range of lattice structures can now be efficiently fabricated at various length scales not previously attainable. SLM is a powder bed process where a laser beam is rasterscanned across a bed of metal powder particles in a specified pattern, layer by layer, to create a 3-dimensional (3D) part. Metal AM methods have opened the design space immensely for building low-density structures with high strength, however these methods can result in structures that vary significantly from the idealized design. Specifically, the powder bed process is known to introduce unwanted defects into a metal structure, such as "parasitic" material, porosity, and surface distortion. Some processing related defects are 
due to issues such as lack of fusion and gas porosity, which are difficult to control and can yield parts with a variety of densities and void distributions [12].

Understanding and predicting the mechanical behavior of lattice structures fabricated in such a manner is therefore important as the intended applications are reliant on the structural integrity of such parts. Many recent studies have focused on such investigations $[6,10,13-21]$, highlighting the role of many factors, such as the microstructure of the metal, defects introduced during the build process, and lattice topology. Attention in particular has focused on the scaling relationship between apparent elastic stiffness and relative density, which is sensitive to the lattice topology [22]. In addition, Mazur et al. [18] and others $[16,23]$ have shown that failure modes and the transition from linear elastic and non-linear anelastic response varies based on whether the lattice is stretch- or benddominated and the relative density of the lattice. Lattice topology thus dictates the stressstrain response; for example, lattice structures with a stretch-dominated topology have exceptional stiffness and strength for a given relative density, while lattice structures with a bend-dominated topology and same relative density show more compliance, significant softening after the onset of anelasticity, and are known to absorb energy well [22]. Also, bend-dominated lattices exhibit a plateau stress similar in magnitude to the initial peak stress.

Along with topology, the microstructure of the selected AM material is an important factor in the mechanical performance. A wide range of possible metals and alloys can be selected and, in turn, the microstructure of each type of metal can be manipulated by applying 
various heat treatments. Often AM metals, like Ti-6Al-4V (Ti64), are heat treated in order to increase ductility [20, 24-26]. Along with microstructure, fabrication defects can, depending on the severity and defect distribution, play a significant role in affecting the mechanical response. Tomography, a 3D non-destructive imaging technique used to glean structural information with micrometer resolution over a several mm field of view $[27,28]$, has played an invaluable role in this regard. Also, in-situ tomography has been used to investigate both defects and failure mechanisms in metals [12, 29-32] by tracking the damage evolution and defect distribution, elegantly showing how large defects can alter the failure mechanisms. Several studies have used tensile testing during X-ray tomography to map void growth in heterogeneous ductile materials, such as dual phase steels [31] and Ti6Al4V [32]. At the Advanced Light Source's (ALS, LBNL, Berkeley, CA) tomography beamline (8.3.2) there is a dedicated custom built mechanical testing device developed by Haboub et al. [33] and Bale et al. [34] that can test structures in both compression or tension. The high flux achieved at synchrotron facilities enables in-situ mechanical testing to take place over only a few hours, while lab based tomography systems would take a prohibitively long time to acquire the necessary timesteps required during loading.

Although there has been significant attention placed on evaluating the stress-strain or force-displacement response of lattices with different material properties, defects, and topologies, most of these studies fall short in understanding the local deformation response. This is a significant void in our understanding, as the derived macroscopic response may not really represent a material point anywhere in the structure considering the stress is not uniformly distributed. To this end, in this study we investigate in situ - 
using high resolution synchrotron radiation micro-tomography (SR $\mu \mathrm{T})$ - the compression response of unit cell lattice structures with two different topologies: octet-truss (OT), which is stretch-dominated, and rhombic dodecahedron (RD), which is bend-dominated. The SR $\mu \mathrm{T}$ provides real time 3D images with micrometer resolution and the tomography data is used to evaluate failure mechanisms, to identify defects in the SLM structure, and to track the localized strain during different amounts of imposed loading. These results are then compared to computational models, finite element and equivalent continuum, developed to predict the elastic and failure behavior of these types of light-weighting structures.

\section{Experimental Procedures}

\subsection{Fabrication of lattice structures}

A total of six different types of Ti64 alloy structures (two different topologies and three different relative densities) were built using a powder bed system (Concept Laser M2) at FineLine Prototyping (Raleigh, NC) ${ }^{1}$. The sample test matrix is shown in Table 1. Before being removed from the build plate, all samples were heat-treated at $900^{\circ} \mathrm{C}$ for 1 hour using a vacuum furnace at a heating rate of $10{ }^{\circ} \mathrm{C} / \mathrm{min}$. After heat-treatment the samples were gas cooled down to room temperature. There were two sets of SLM Ti64 lattice samples built at different times, which were heat-treated in different furnaces. It should be noted that these two batches of samples displayed different mechanical properties; this could be due to the subsequent build and processing differences. The material model used here was tuned to results from the OT 10\% relative density unit cell (from the second batch

\footnotetext{
${ }^{1} \mathrm{http} / / /$ www.finelineprototyping.com/
} 
of samples) and then used for all other cases within that sample group. It is important that the model is tuned to the correct sample group since consequently if the mechanical properties of the material being tested are inaccurate then the entire model predictions will be incorrect.

The connectivity of the struts and the shape of the unit cell define the lattice topology. The unit cell dimensions for the two different lattice topologies, OT and RD, were selected in order to reach a target relative density using sub-millimeter strut diameters. The selected relative densities were 10,20 , and $30 \%$, where the relative density is defined by the ratio of the macroscopic density of the cellular structure to the density of the structure's material:

$$
\bar{\rho}=\frac{\rho}{\rho_{S}}
$$

Each topology's relative density is defined below by the following approximate analytical relationships:

$$
\begin{aligned}
& \bar{\rho}_{O T}(a, l)=6 \sqrt{2} \pi\left(\frac{a}{l}\right)^{2} \\
& \bar{\rho}_{R D}(a, l)=\frac{3}{2} \sqrt{3} \pi\left(\frac{a}{l}\right)^{2}
\end{aligned}
$$

Where $a$ and $l$ are the radius and length of a strut, respectively [17]. 


\subsection{Compression Testing and Tomography}

The in-situ compression tests were performed on unit cell lattices in a custom built testing fixture designed to fit within the tomography hutch and allow for $180^{\circ}$ rotation. The tomographic imaging was performed at Beamline 8.3.2 at the Advanced Light Source (Lawrence Berkeley National Laboratory, Berkeley, CA, USA). The experimental setup was similar to standard tomographic procedures [35] where the sample and testing rig were rotated in an X-ray beam and the transmitted radiographic projections were imaged via a scintillator, magnifying lens, and a digital camera. For this experiment the effective voxel size was $3.3 \mu \mathrm{m}$. The samples were mainly imaged in polychromatic or 'white' light mode, where the entire available energy spectrum is used. This mode is useful when scanning high-density metals. Reconstructed images were obtained via a filtered back-projection algorithm using the software package Octopus [36]. Three-dimensional visualization, segmentation, and quantification was performed using Avizo ${ }^{\mathrm{TM}}$ software [37].

During compression testing each lattice structure was loaded using displacement control at a nominal quasi-static strain rate of $10^{-3} \mathrm{~s}^{-1}$. During testing the lattice was held at specified displacements along the force-displacement curve to allow for the entire tomography scan to complete, with each scan taking $\sim 5$ minutes. There was some relaxation observed during each scan.

\subsection{Tomography analysis, quantifying local strain in individual struts}

Tomography was used to track the location of each of the structure's nodes in 3D space through time during loading. The initial tomographic dataset for each unit cell possessed a 
full geometric description of each sample, however the reconstructed images were much more complex than the description used in a finite element model of the idealized sample. It is therefore convenient to refine further the tomographic reconstructions to facilitate direct comparison with models of the idealized sample. Specifically, each of the reconstructed tomography datasets were transformed into a more simplified graph description that directly matches the input and output of the finite element model. The outcome of this process results in the ability to measure local ligament-average strains directly from the tomographic datasets.

For segmentation of the tomography data, each dataset was converted to a 3D binary volume by a single threshold value. Pores and cracks were then removed through a morphological close operation. For each of the material points, the distance to the nearest non-material (air) point was calculated. Voxels on the surface have small values (1) while voxels in the center of nodes and ligaments have larger ones. We then removed $>90 \%$ of the remaining volume voxels according to the calculated distances; we kept only those voxels furthest away from the surface. This has the effect of producing a thinned version of the initial dataset. Then for each of the connected components left we defined their centerof-mass position and calculate the minimum distance from each node to every other node. The mean distance, calculated for each node, was then defined as the average strut length. At this point we can calculate the average micro-strain in each strut using the location of all of the nodes in the system. 


\section{Results and Discussion}

\subsection{Deformation response}

This study investigates the real-time 3D deformation behavior of OT and RD unit cell structures by tracking both micro- and macro-scale deformation simultaneously. As previously mentioned, each 3D tomographic dataset provides information on the as-built structure and the 3D spatial location of each node and strut (ligaments connecting the nodes), which is tracked through time to quantify localized strain and identify failure mechanisms. For each type of sample tested (Table 1) the force versus displacement is recorded and the results are shown in Figure 1. Additional compression tests were performed on the same types of lattice structures in order to validate the in-situ testing setup and to provide more statistics on the mechanical response for each of the 6 types of lattice structures studied here. Figure 2 shows the force-displacement results from these additional tests. The additional compression tests show a similar trend in mechanical properties, specifically maximum load and force versus displacement behavior, for each sample type. The similarities in results demonstrate that, even though there are large variations between the as-built additively manufactured structures (i.e. defects, such as surface roughness, porosity distribution, strut thickness variation), the overall macro-scale mechanical behavior is shown to be similar for samples built with the same target structure (relative density, topology, and parent material) and built on the same SLM build. However, close attention should still be placed on variations between builds and heat treatments. Indeed, the density and defect distribution should always be considered and compared to the idealized case. 
As expected, the force-displacement curves in both Figures 1 and 2 show that there is a difference in the transition from linear elastic to non-linear anelasticity for samples with different topologies, OT versus RD. Specifically, there is a much more abrupt transition for the stretch-dominated lattice (OT), while the transition is much smoother for the benddominated lattice (RD). Also the force-displacement curves show an increase in stiffness and strength as relative density increases for samples with the same topology. Overall there are major differences in mechanical properties observed between different lattice structures depending on the microstructure, relative density, and topology, which all need to be considered when designing a light-weighting structure.

The tomography results are used to evaluate failure modes for each type of structure and these results are useful for explaining differences in mechanical properties. A time series of 3D tomographs accompany the macro-scale deformation responses and an example of these results is shown in Figure 3. Figure 3a shows a representative force-time curve for one of the samples tested (10\% relative density OT unit cell lattice structure) and Figure $3 \mathrm{~b}$ shows the corresponding force-displacement curve. The images in Figure 3c are 3D surface images of the sample rendered from the reconstructed tomography data: image 1) shows the 3D surface prior to loading and image 2) shows the same sample after it starts to anelastically deform. Although there are only two 3D images shown here, every pause in the force-time graph (Fig. 3a) represents an acquisition of a tomographic dataset. 
The experimental results capture each sample's structural response under a specific loading and displacement condition. This allows for tracking location and degree of deformation occurring in individual struts. Figure 4 shows a comparison between an RD and OT both with $20 \%$ relative density. The numbers indicate the displacement values at which the tomography images are acquired. Here it is shown that the deformation is much more uniform in the RD sample (Fig. 4a - Images \#3 \& 4) and more heterogeneous in the OT sample (Fig. 4b - Image \#3). For the OT structure, struts that are slimmer, located on the outside, and experience compressive loading start to deform first via buckling (Fig. 3b Image 3).

The failure modes are assessed by segmenting out certain struts within the structure to identify the degree of deformation. An example of this is shown in Figure 5, where the 10\% relative density structures are shown during elastic and non-linear anelastic deformation and certain regions of the structure are cropped to compare regions where larger deformation occurs. Here specific struts in the OT structure (Fig. 5b) have started buckling, while the RD structure (Fig. 5a) displays more of a uniform crushing. Also it was observed that, for the $10 \%$ OT structure, the degree of buckling in each strut is not homogenous and depends on the orientation of the strut as well as the degree of defects in the as-built strut.

The tomography data is analyzed further by generating individual graphs mapping localized strain in the struts for each load step, where the Euclidean distance between each node's centerline is measured and then compared to the unloaded Euclidean distance, in order to quantify localized strain along each strut. As discussed in the previous section, a 
refinement of node position is implemented. This is absolutely necessary if we are to measure changes in ligament length within the material's elastic limits (fractions of a percent sensitivity required). Through this analysis a 3D image registration of each node position during deformation is determined. The error in this registration technique was tested and found to be on the order of $10^{-2}$ pixel for exact shape matching. The relative strain is then determined and compared to the strain predicted using the finite element model. Figure 6 depicts the process of reducing the data from a node (shown in Fig. 6b) to (a) an edge map, (c) a spatial map connecting nodes. The reduced map showing the connectivity is compared to the unloaded state to produce the localized ligament average strain over time (d), where each scan number corresponds to increasing load. The colors in Fig. 6d correspond to individual struts in the lattice. This reduced map is useful for comparing the experimental results to the simulation predictions, which will be discussed in the next section.

\subsection{Modeling methods - Deformation predictions and comparison to experimental results}

Two different computational studies are implemented here with the goal of predicting the deformation behavior of each type of lattice structure at different length-scales. Specifically, the finite element model is used to model the micro- and macro- strain response of the lattice structures and the predictions are compared to the experimental results, while an equivalent continuum model [24] is utilized to predict failure modes in stretch-dominated lattices and these predictions are validated using the tomography results. 


\subsubsection{Predictive meso- scale deformation behavior, finite element model response}

The finite element model of a lattice unit cell, which is a detailed representation composed of hundreds of thousands of 3D hexahedral elements, is used to model the deformation of the unit cell. Analysis is performed using LLNL's in-house numerical simulation software tool ALE3D, which in these calculations is run in quasi-static mode with implicit time stepping. A Johnson-Cook strength material model is used to model post-yielding behavior of the material. Then a suite of tensile tests performed on bulk SLM material is used to fit the necessary parameters for the material model. The bottom face sheet of the unit cell is constrained in all directions while the top face sheet, which, is being displaced, is allowed to move in all directions.

A comparison between the finite element model predictions and the experimental results, from the additional compression tests, is shown in Figure 7, which presents the forcedisplacement curves for both the simulation and experiment for all six types of structures. There is a relatively good match here; the only major deviation is observed for the OT $30 \%$ relative density and RD 10\% relative density. The deviation in the $10 \% \mathrm{RD}$ can likely be explained by the fact that the as-fabricated sample is over built, so it has a higher relative density than the target $10 \%$. The deviation in $30 \%$ OT is initially unknown, but can be investigated further by analyzing that sample's tomography data. Along with the forcedisplacement predictions, the finite element model is also used to predict the micro-strain field in the lattice during deformation, which is compared to the node tracking tomography analysis. 
Figure 8 (a)-(c) compares the force-displacement curves for two separate experiments (taken from the in situ tomography and the additional compression tests) and the simulation for a representative OT with three different relative densities: 10\% (Fig. 8a), 20\% (Fig. 8b), and 30\% (Fig. 8c). Figure 8 (d) \& (f) plots the relationship between the strains in the struts versus loading for representative OT (10, and $30 \%$ relative density) structures. For the plots in Fig. 8 (d) \& (f) each line in the plot represents a different strut with the solid and shaded lines corresponding to the simulation and experimental results, respectively. The red lines are struts in tension, while the blue and green lines are struts in compression. As expected, as the load increases, strains increase. For the octet $10 \%$ case, seen in Figure 3d, the experimental strain results could only be generated for the struts in tension due to limitations in the tomography setup for that sample.

Figure 8e presents the macro-displacement versus the micro-strain in the struts for the $20 \%$ OT, this is plotted on different axes to show what happens after the transition out of the elastic region, the line defining the elastic region is determined by the displacement at the yield strength (assuming a $0.2 \%$ offset), where it is observed that the strain in the struts begins to go non-linear prior to this transition point (shown in yellow in plot Fig 8e). This deviation from linearity is of interest since this could indicate a change in failure mode, which is thought to change around $20 \%$ relative density in OT structures.

The force-displacement curve compares well between the experiment and simulation so it is not surprising that the localized strain results are generally in agreement. The increase in 
tensile strain in the in-plane ligaments with overall compressive loading agrees phenomenologically with the behavior expected. It is important to note that the simulation model is an idealized representation of the unit cell with exact dimensioning given to the strut diameter and strut length. Due to some deviations and imperfections in the as-built unit, the experimental results have some more deviation in strain levels among nominally equivalent struts but the grouping remains consistent with the simulation and with the increase in loading.

While Fig. 8 shows that the force-displacement curves for the $10 \%$ and $20 \%$ OT are in good agreement, the $30 \%$ relative density case gives a significant difference in the stiffness when comparing the simulation and experiment. Also, there is a kink observed in the macro-scale response for the $30 \%$ relative density OT case. It is unclear why this occurred upon initial inspection of the test results, but the localized strain comparison clearly shows a significant deviation in the strut's strains. Some of the diagonal struts (dotted lines in Fig. 8f), which should be in compression, are in tension initially but then, as load increases, they undergo compressive strains that are similar to the simulation at the highest load levels. This could be explained by residual stress or defects that cause a non-uniform distribution of load, which lead to the different response in the stiffness of the force-displacement curve. The differences seen in the other struts can be explained by load redistribution due to the transfer of load from the off-nominal struts.

Figure 9 maps the localized strain for individual struts in the 30\% OT structure (under $\sim 850 \mathrm{~N}$ of load) using the simulation predictions and the experimental results; the plots 
help to highlight which struts in the unit cells have noticeable differences in strain. It becomes obvious looking at the two structures that there are a few diagonal struts of the experimental unit cell in the upper right corner that are not undergoing significant strain. The utility of this analysis method is highlighted by this particular case where a deviation of the force-displacement curve is observed for the $30 \%$ relative density OT but there is no way of understanding the difference between the results without an understanding of the as-built structure. Here the strut strain analysis method identifies the particular struts that may be causing the difference between the cases, as well as the how the strains evolve with loading. This also highlights how robust the structures can be, especially when the material selected is also robust, so that it allows for load redistribution among struts before buckling or fracture occurs. Overall, although the deformation response is inhomogeneous, the max load seen by this structure remains similar to the predicted value and overall there is not a huge difference in the macro-scale response.

As was demonstrated in Figure 7 and 8, the general agreement with the finite element model and experimental results is relatively good. However, there is some variation in the predictions and the model, especially for 30\% (Fig. 7-9), and this is due at least in part to the fact that the finite element models used here do not take into account defects in the asbuilt structure, which can be characterized using the tomography data. Specifically, deviations between the model prediction and experimental results for each individually measured ligament strain are likely due to variation in the additive process of building individual ligaments. A better match with the model could be achieved if effective ligament diameters were all fed in as the initial starting point of the model. Currently the model 
assumes an idealized structure with no ligament thickness or cross-sectional area variation, however these area variations occur in all the as-built lattice structures. This assumption imposes symmetry in the system effectively precluding the simulation from calculating any difference in strain for any of the in-plane ligaments.

3.2.2. Validating the equivalent continuum model predictions with experimental findings, failure modes in lattice structures

As described in [38], the equivalent continuum analysis assumes the structure deforms elastically up to some limit stress. The model calculates this limit stress by examining the elastic distribution of forces in the struts of a periodic structure under some macro-scale applied stress field. Given this distribution of forces, the method determines the applied stress at which the first strut undergoes inelastic deformation. Depending on the underlying material response this applied stress can be the macro-scale limit stress.

This methodology uses an interaction equation to define strut failure based on the elastic forces in each strut. Figure 10 shows results using an interaction equation based on the AISC Steel design manual [39] considering the interaction of two strut failure modes: plastic yielding and structural instability (buckling). The inputs to this interaction equation are the forces applied to the strut, the strut cross-sectional geometry, strut material properties -- in particular the material Young's modulus and yield stress, and a parameter $\mathrm{K}$-- a column effective length factor -- describing the relative stiffness of the joints. Here $\mathrm{K}$ $=0.5$ represents ideally rotationally rigid joints and $\mathrm{K}=1.0$ represents ideally pinned joints. The actual joints in the as-build structure fall somewhere between these two 
extremes.

Figure 10 plots the relative density of a periodic structure versus the structure's limit stress for uniaxial macro-scale loading for three different values of K: 0.5, 0.65, and 1.0. For the models with stiffer joints $(K=0.5$ and 0.65$)$ the plot shows an inflection point indicating a transition between a buckling-dominated failure mechanism and failure by full-section plastic yielding. For $\mathrm{K}=1.0$ the model predicts buckling-dominated failure for all relative densities between $5 \%$ and $30 \%$.

The equivalent continuum model indicates a clear transition between buckling- and yielddominated failures as the relative density of the structure increases. However, this critical relative density depends on the rigidity of the joints, which will be strongly influenced by the manufacturing process. Based on the tomography and experimental analyses this transition is likely somewhere between 10 and 20\% relative density for the OT structure.

This tradeoff is important because the failure mechanism (buckling versus yielding) strongly influences the ductility of the structure. A yield-dominated structure tends to have better ductility than a buckling-dominated structure. For work hardening materials, full section yielding in one strut does not immediately lead to the collapse of the periodic structure, whereas a periodic buckling mechanism might immediately cause collapse or at least significant deformation to some snap-through configuration.

The predicted buckling/yielding transition point depicted in Figure 10 can be validated 
using in situ tomography and mechanical testing. Specifically, there is a noticeable change in the force-displacement behavior, both experimentally and with finite element model predictions (Fig. 7), for structures with 10 and 20\% relative density. For the $10 \%$ OT structure there is a much sharper transition between linear elastic and non-linear anelasticity, where the load is shown to decrease much more quickly, than is seen in the 20 and $30 \%$ relative density structures. This sharp decrease is indicative of buckling in the struts. This assumption is further validated by looking at the tomography images of the deformed 10\% OT structure, where buckling is observed to occur (Figure 3 - Image 2) immediately following the onset of anelasticity. For the $20 \%$ and $30 \%$ lattices (during continuous strain) the load holds steady before decreasing again. Recall also that Figure 8e suggests that the trend for micro-strain in the struts goes non-linear relatively early in the loading history. Together, these observations indicate that for 20\% OT structures the struts begin deforming by plastic yielding followed later by buckling, which was shown (Figure 4b - Image 3) to eventually occur as strain in the lattice continues.

\section{Conclusion}

AM technology enables the manufacturing of light-weighting lattice structures with a wide range of possible densities and properties. This leads to many competing designs, so there is a need for simulations that can accurately predict the mechanical behavior of these complex structures and that take into account defects, relative density, topology, and microstructure. This study evaluated the local strain behavior in unit cell lattices with different topologies and relative densities and compared the results to two predictive failure models. The findings presented in this study demonstrate the following conclusions: 
1. In-situ tomography was used to map localized strain across struts and the results showed that the deformation response for the stretch dominated lattice, OT, was heterogeneous. The strain map identified certain struts that were not undergoing significant strain, while other struts were found to be over compensating with higher strain than predicted using a finite element model. Although the micro-strain predictions varied from the experimental results, the finite element model was fairly accurate at predicting the experimental force-displacement results for the unit cell lattice structures tested here. This showed that even though there were defects in the as-built structure, the lattices were fairly good at distributing the load in order to avoid early catastrophic failure.

2. The equivalent continuum model predicted a transition in failure mode from buckling to yielding for stretch dominated lattices. This was shown, experimentally, to occur between 10 and $20 \%$ in the OT. Specifically, the in-situ tomography data showed evidence of buckling occurring very early in the $10 \%$ OT structure, while there was a significant change in force-displacement behavior observed between the 10 and $20 \%$ OT.

As a result of these analyses we have begun to incorporate this variation into our modeling framework to understand and predict variations of macro-mechanical respond due to variation on the micro-scale. This feedback loop between modeling and experiment may enable efforts in the future aimed at uncertainty quantification. 


\section{Acknowledgements:}

This work was performed under the auspices of the U.S. Department of Energy by Lawrence Livermore National Laboratory under Contract DE-AC52-07NA27344. The Advanced Light Source is supported by the Director, Office of Science, Office of Basic Energy Sciences, of the U.S. Department of Energy under Contract No. DE-AC0205CH11231. The authors would like to thank Drs. Alastair MacDowell and Dula Parkinson for their outstanding support of this experimental work. We would like to acknowledge Ray Swan for providing the designs for the modified base of the test rig.

Table:

\begin{tabular}{lccc}
\hline Topology & $\begin{array}{c}\text { Target Relative } \\
\text { Density } \\
\mathbf{( \% )}\end{array}$ & $\begin{array}{c}\text { Strut } \\
\text { diameter } \\
\mathbf{( m m )}\end{array}$ & $\begin{array}{c}\text { Strut length } \\
\mathbf{( m m )}\end{array}$ \\
\hline $\mathrm{RD}$ & 10 & 0.37 & 1.67 \\
$\mathrm{RD}$ & 20 & 0.52 & 1.67 \\
$\mathrm{RD}-$ & 30 & 0.64 & 1.67 \\
\hline OT & 10 & 0.37 & 3.02 \\
OT & 20 & 0.52 & 3.02 \\
OT & 30 & 0.64 & 3.02 \\
\hline
\end{tabular}

$\mathrm{RD}=$ Rhombic dodecahedron, OT $=$ Octet truss

Table 1. Presents the test matrix for the in situ tomography experiments. 
References:

[1] I. Maskery, A.O. Aremu, M. Simonelli, C. Tuck, R.D. Wildman, I.A. Ashcroft, R.J.M. Hague. Mechanical Properties of Ti-6Al-4V Selectively Laser Melted Parts with BodyCentred-Cubic Lattices of Varying cell size, Exp Mech 55 (2015) 1261-1272.

[2] J. Xiong, R. Mines, R. Ghosh, A. Vaziri, L. Ma, A. Ohrndorf, H.-J. Christ, L. Wu. Advanced Micro-Lattice Materials, Advanced Engineering Materials 17 (2015) 1253-1264.

[3] R.A.W. Mines. On the Characterisation of Foam and Micro-lattice Materials used in Sandwich Construction, Strain 44 (2008) 71-83.

[4] M.G. Rashed, M. Ashraf, R.A.W. Mines, P.J. Hazell. Metallic microlattice materials: A current state of the art on manufacturing, mechanical properties and applications, Materials \& Design 95 (2016) 518-533.

[5] C. Yan, L. Hao, A. Hussein, P. Young, D. Raymont. Advanced lightweight 316L stainless steel cellular lattice structures fabricated via selective laser melting, Materials \& Design 55 (2014) 533-541.

[6] J. Kadkhodapour, H. Montazerian, A.C. Darabi, A.P. Anaraki, S.M. Ahmadi, A.A. Zadpoor, S. Schmauder. Failure mechanisms of additively manufactured porous biomaterials: Effects of porosity and type of unit cell, Journal of the Mechanical Behavior of Biomedical Materials 50 (2015) 180-191.

[7] L.E. Murr, S.A. Quinones, S.M. Gaytan, M.I. Lopez, A. Rodela, E.Y. Martinez, D.H. Hernandez, E. Martinez, F. Medina, R.B. Wicker. Microstructure and mechanical behavior of Ti-6Al-4V produced by rapid-layer manufacturing, for biomedical applications, Journal of the Mechanical Behavior of Biomedical Materials 2 (2009) 20-32.

[8] M.N. Ahsan, C.P. Paul, L.M. Kukreja, A.J. Pinkerton. Porous structures fabrication by continuous and pulsed laser metal deposition for biomedical applications; modelling and experimental investigation, Journal of Materials Processing Technology 211 (2011) 602609.

[9] P. Rochus, J.Y. Plesseria, M. Van Elsen, J.P. Kruth, R. Carrus, T. Dormal. New applications of rapid prototyping and rapid manufacturing (RP/RM) technologies for space instrumentation, Acta Astronautica 61 (2007) 352-359.

[10] R. Gümrük, R.A.W. Mines, S. Karadeniz. Static mechanical behaviours of stainless steel micro-lattice structures under different loading conditions, Materials Science and Engineering: A 586 (2013) 392-406.

[11] P. Li. Constitutive and failure behaviour in selective laser melted stainless steel for microlattice structures, Materials Science and Engineering: A 622 (2015) 114-120.

[12] H.D. Carlton, A. Haboub, G.F. Gallegos, D.Y. Parkinson, A.A. MacDowell. Damage evolution and failure mechanisms in additively manufactured stainless steel, Materials Science and Engineering: A 651 (2016) 406-414.

[13] S. Merkt, C. Hinke, J. Bültmann, M. Brandt, Y.M. Xie. Mechanical response of TiAl6V4 lattice structures manufactured by selective laser melting in quasistatic and dynamic compression tests, J. Laser Appl. 27 (2015) S17006.

[14] A. Hussein, L. Hao, C. Yan, R. Everson, P. Young. Advanced lattice support structures for metal additive manufacturing, Journal of Materials Processing Technology 213 (2013) 1019-1026.

[15] C. Qiu, S. Yue, N.J.E. Adkins, M. Ward, H. Hassanin, P.D. Lee, P.J. Withers, M.M. Attallah. Influence of processing conditions on strut structure and compressive properties 
of cellular lattice structures fabricated by selective laser melting, Materials Science and Engineering: A 628 (2015) 188-197.

[16] S. McKown, Y. Shen, W.K. Brookes, C.J. Sutcliffe, W.J. Cantwell, G.S. Langdon, G.N. Nurick, M.D. Theobald. The quasi-static and blast loading response of lattice structures, International Journal of Impact Engineering 35 (2008) 795-810.

[17] V.S. Deshpande, N.A. Fleck, M.F. Ashby. Effective properties of the octet-truss lattice material, Journal of the Mechanics and Physics of Solids 49 (2001) 1747-1769.

[18] M. Mazur, M. Leary, S. Sun, M. Vcelka, D. Shidid, M. Brandt. Deformation and failure behaviour of Ti-6Al-4V lattice structures manufactured by selective laser melting (SLM), Int J Adv Manuf Technol (2015) 1-21.

[19] R. Wauthle, B. Vrancken, B. Beynaerts, K. Jorissen, J. Schrooten, J.-P. Kruth, J. Van Humbeeck. Effects of build orientation and heat treatment on the microstructure and mechanical properties of selective laser melted Ti6Al4V lattice structures, Additive Manufacturing 5 (2015) 77-84.

[20] B. Gorny, T. Niendorf, J. Lackmann, M. Thoene, T. Troester, H.J. Maier. In situ characterization of the deformation and failure behavior of non-stochastic porous structures processed by selective laser melting, Materials Science and Engineering: A 528 (2011) 7962-7967.

[21] M. Suard, G. Martin, P. Lhuissier, R. Dendievel, F. Vignat, J.J. Blandin, F. Villeneuve. Mechanical equivalent diameter of single struts for the stiffness prediction of lattice structures produced by Electron Beam Melting, Additive Manufacturing 8 (2015) 124-131.

[22] M.F. Ashby. The properties of foams and lattices, Philosophical Transactions of the Royal Society A: Mathematical, Physical and Engineering Sciences 364 (2006) 15-30.

[23] T. Tancogne-Dejean, A.B. Spierings, D. Mohr. Additively-manufactured metallic micro-lattice materials for high specific energy absorption under static and dynamic loading, Acta Materialia 116 (2016) 14-28.

[24] F. Brenne, T. Niendorf, H.J. Maier. Additively manufactured cellular structures: Impact of microstructure and local strains on the monotonic and cyclic behavior under uniaxial and bending load, Journal of Materials Processing Technology 213 (2013) 15581564.

[25] S. Leuders, M. Thöne, A. Riemer, T. Niendorf, T. Tröster, H.A. Richard, H.J. Maier. On the mechanical behaviour of titanium alloy TiAl6V4 manufactured by selective laser melting: Fatigue resistance and crack growth performance, International Journal of Fatigue 48 (2013) 300-307.

[26] R.P. Mulay, J.A. Moore, J.N. Florando, N.R. Barton, M. Kumar. Microstructure and mechanical properties of Ti-6Al-4V: Mill-annealed versus direct metal laser melted alloys, Materials Science and Engineering: A 666 (2016) 43-47.

[27] T.B. Kim, S. Yue, Z. Zhang, E. Jones, J.R. Jones, P.D. Lee. Additive manufactured porous titanium structures: Through-process quantification of pore and strut networks, Journal of Materials Processing Technology 214 (2014) 2706-2715.

[28] S. Van Bael, G. Kerckhofs, M. Moesen, G. Pyka, J. Schrooten, J.P. Kruth. Micro-CTbased improvement of geometrical and mechanical controllability of selective laser melted Ti6Al4V porous structures, Materials Science and Engineering: A 528 (2011) 7423-7431.

[29] F. Beckmann, R. Grupp, A. Haibel, M. Huppmann, M. Nöthe, A. Pyzalla, W. Reimers, A. Schreyer, R. Zettler. In-Situ Synchrotron X-Ray Microtomography Studies of Microstructure 
and Damage Evolution in Engineering Materials, Advanced Engineering Materials 9 (2007) 939-950.

[30] E. Maire, O. Bouaziz, M. Di Michiel, C. Verdu. Initiation and growth of damage in a dual-phase steel observed by X-ray microtomography, Acta Materialia 56 (2008) 49544964.

[31] C. Landron, E. Maire, O. Bouaziz, J. Adrien, L. Lecarme, A. Bareggi. Validation of void growth models using X-ray microtomography characterization of damage in dual phase steels, Acta Materialia 59 (2011) 7564-7573.

[32] L. Lecarme, E. Maire, A. Kumar K.C, C. De Vleeschouwer, L. Jacques, A. Simar, T. Pardoen. Heterogenous void growth revealed by in situ 3-D X-ray microtomography using automatic cavity tracking, Acta Materialia 63 (2014) 130-139.

[33] A. Haboub, H.A. Bale, J.R. Nasiatka, B.N. Cox, D.B. Marshall, R.O. Ritchie, A.A. MacDowell. Tensile testing of materials at high temperatures above $1700^{\circ} \mathrm{C}$ with in situ synchrotron X-ray micro-tomography, Rev. Sci. Instrum. 85 (2014) 083702.

[34] H.A. Bale, A. Haboub, A.A. MacDowell, J.R. Nasiatka, D.Y. Parkinson, B.N. Cox, D.B. Marshall, R.O. Ritchie. Real-time quantitative imaging of failure events in materials under load at temperatures above $1,600^{\circ} \mathrm{C}$, Nat Mater 12 (2013) 40-46.

[35] J.H. Kinney, M.C. Nichols. X-Ray Tomographic Microscopy (XTM) Using Synchrotron Radiation, Annual Review of Materials Science 22 (1992) 121-152.

[36] D.M. Vlassenbroeck J, Masschaele B, Cnudde V, Van Hoorebeke L, Jacob P. Software tools for quantification of x-ray microtomography at the 'Center for X-ray Tomography' at Ghent University. Nucl Instru Meth Physics Res A 2007. p.442-445.

[37] Avizo $^{\mathrm{TM}}$ 3D visualization framework. Chelmsford, MA.: Mercury CS, 2008.

[38] M.C. Messner, M.I. Barham, M. Kumar, N.R. Barton. Wave propagation in equivalent continuums representing truss lattice materials, International Journal of Solids and Structures 73-74 (2015) 55-66.

[39] A.I.o.S. Construction. Steel Construction Manual, 14th edition, 2011. 


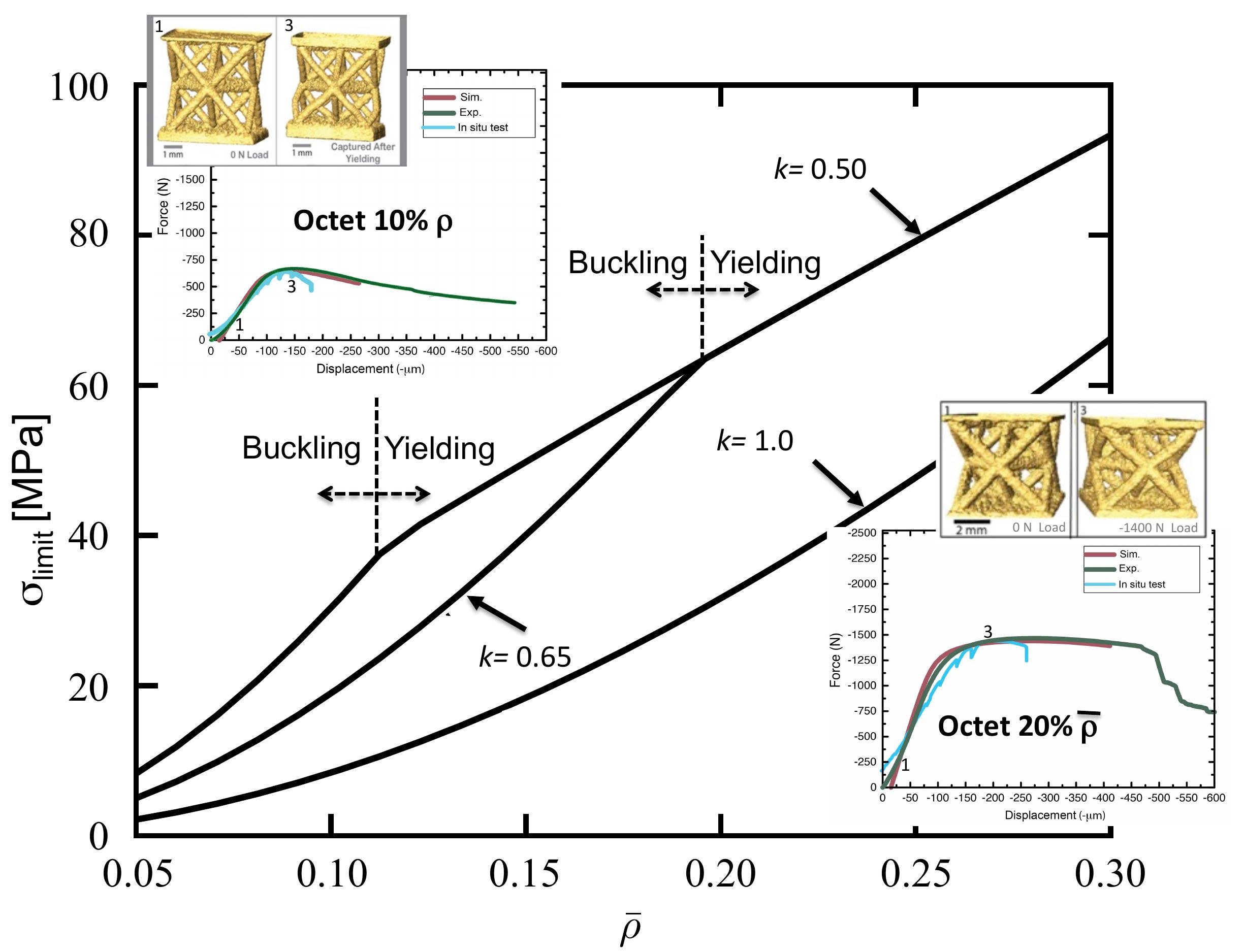



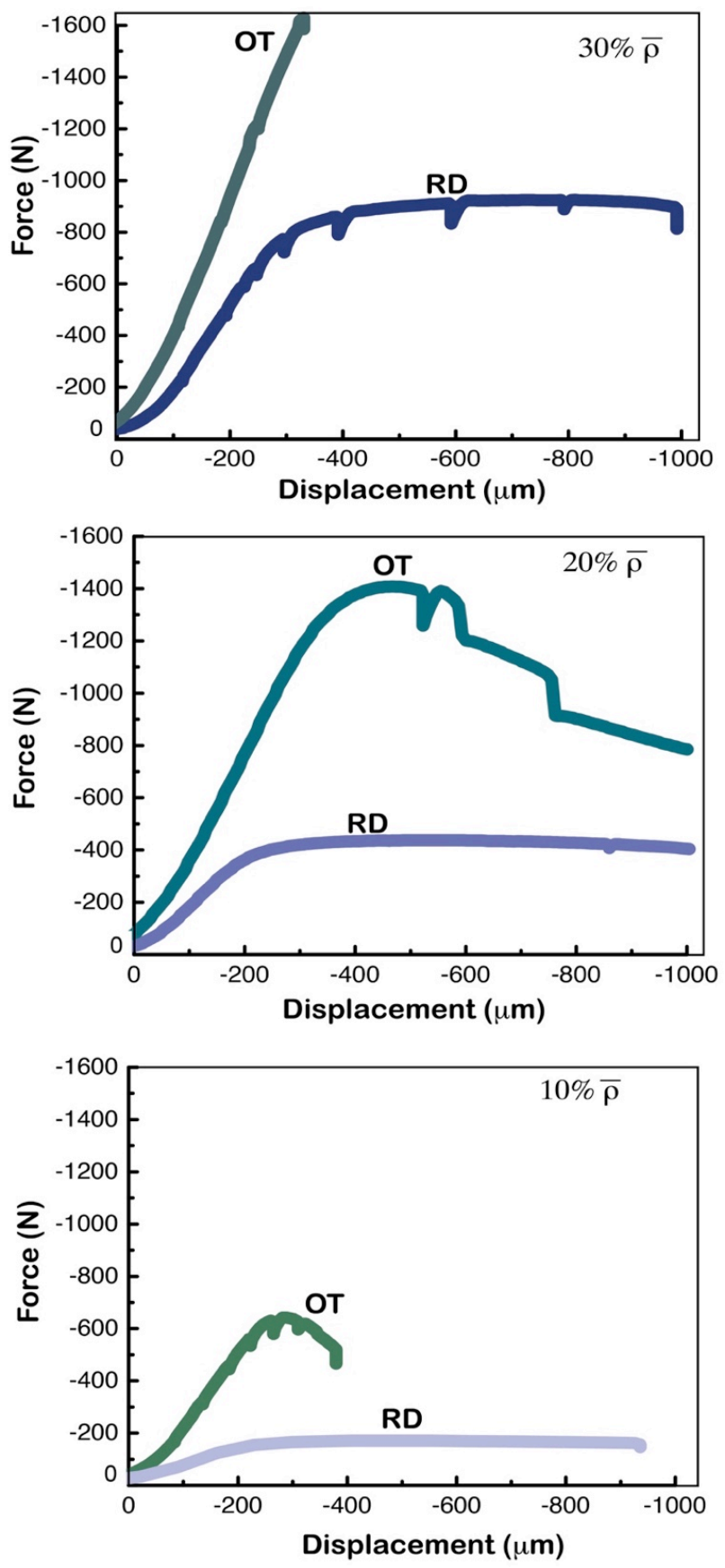

Figure 1. Results from in situ compression tests. Plots comparing the load versus displacement results for octet-truss (OT) and rhombic dodecahedron (RD) unit cell lattice structures with 10,20 , and $30 \%$ relative density tested using in situ compression tests coupled with Synchrotron Radiation micro-Tomography. 

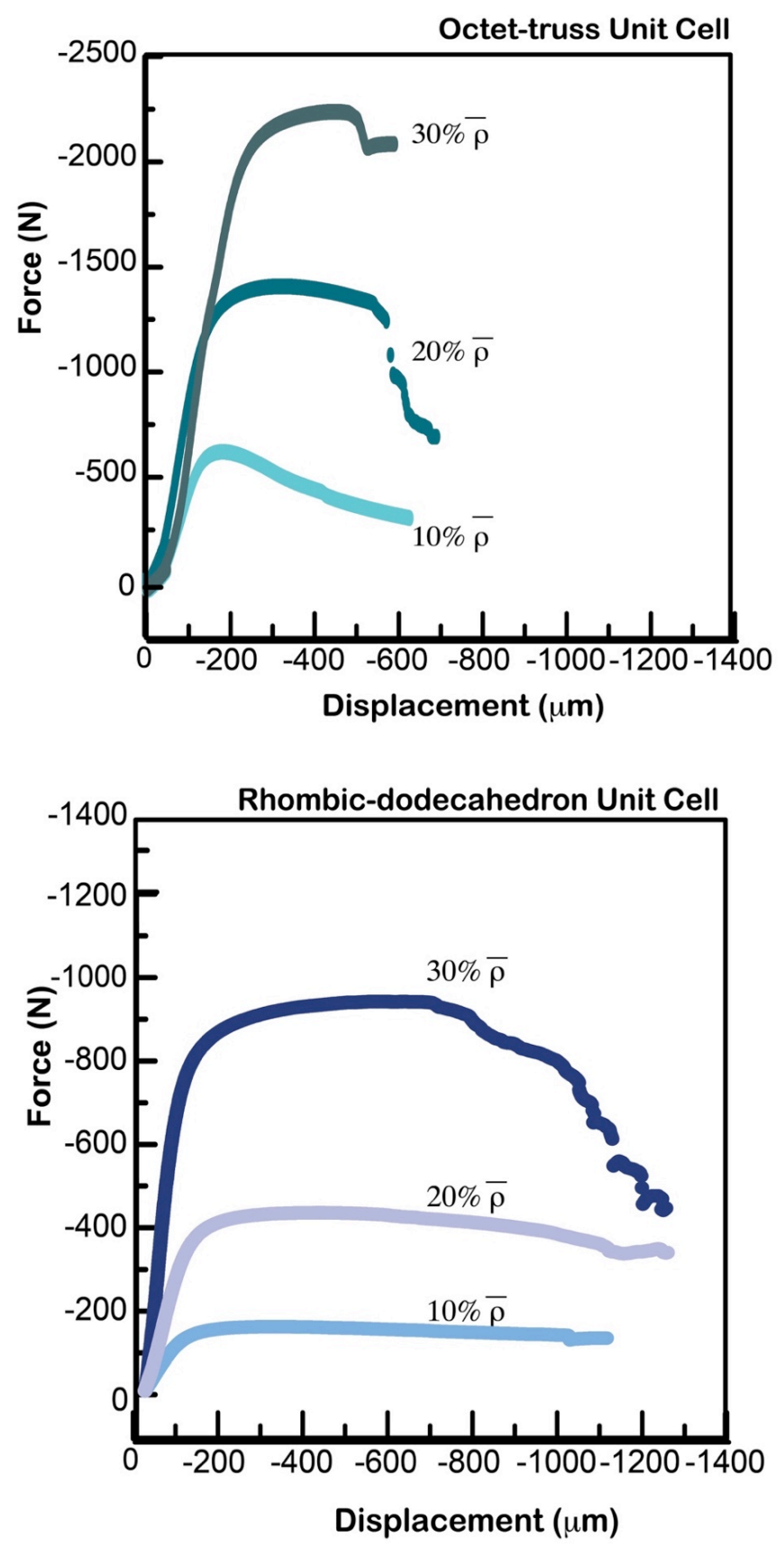

Figure 2. Results from additional compression tests. Force versus displacement for the 6 different types of unit cell lattice structures investigated. Plots compare the results from lattice structures with 10,20 , and $30 \%$ relative density, respectively, for both octet (top plot) and RD (bottom plot). 
a)

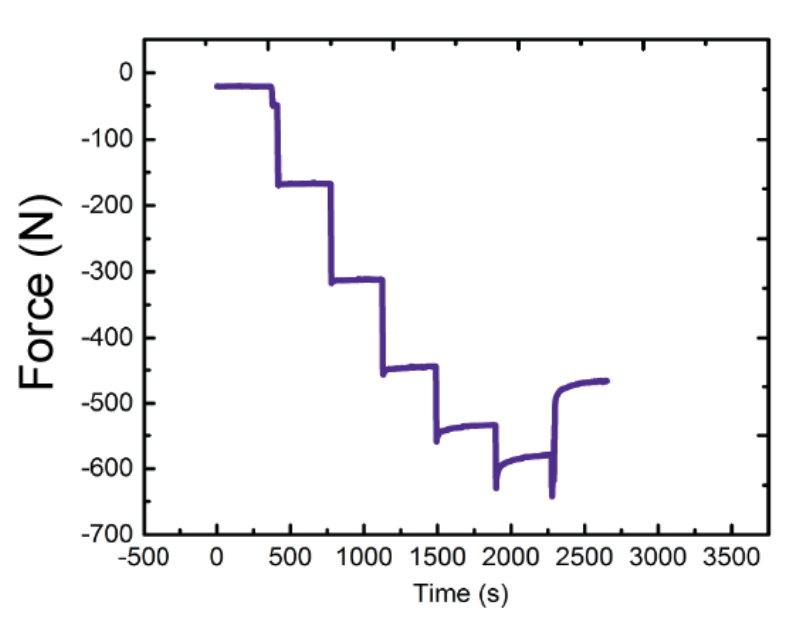

c)
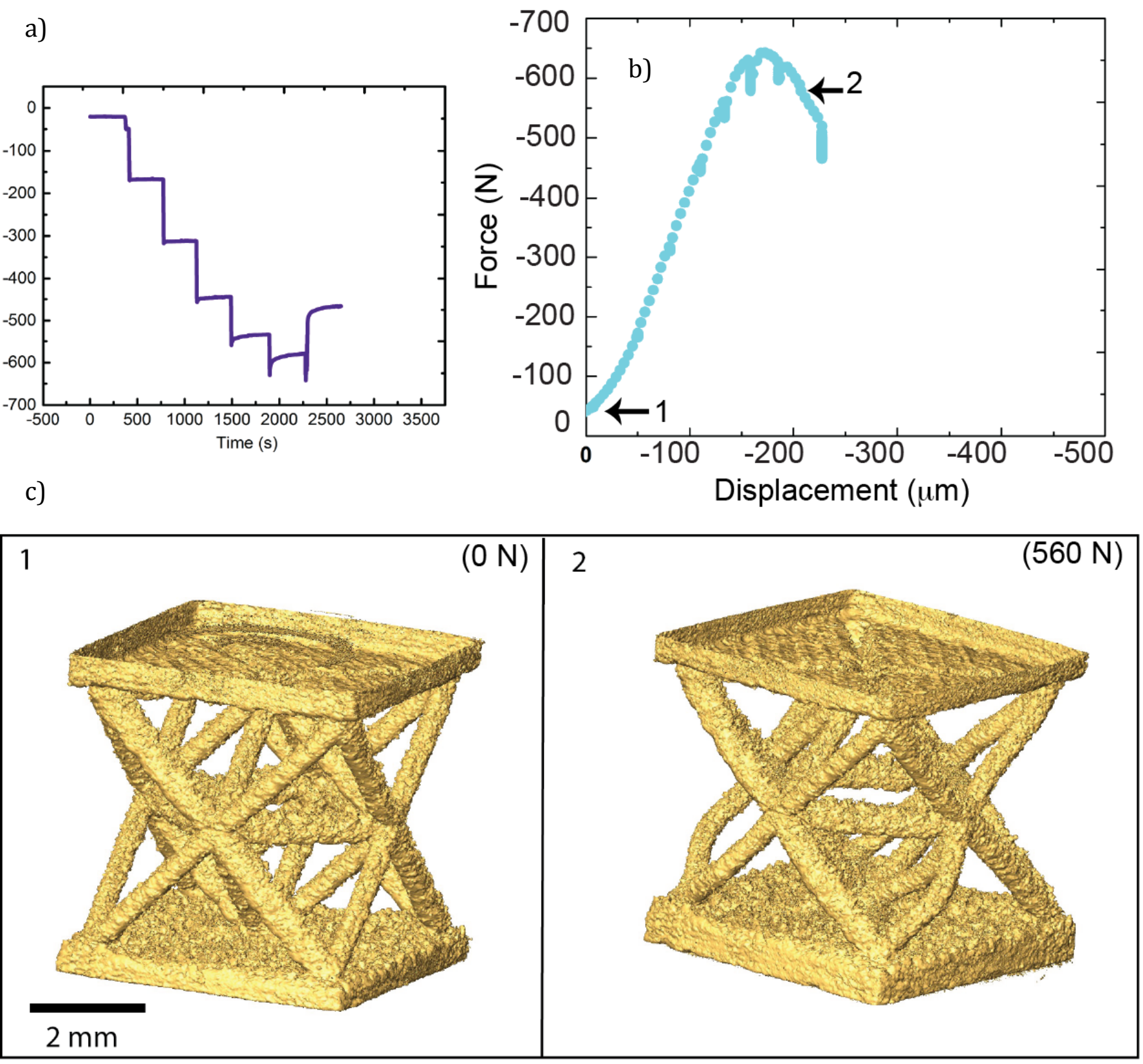

Figure 3. In situ compression results for a $10 \%$ octet structure. a) Showing a representative load versus time data for a $10 \%$ relative density octet unit cell structure, each pause in the load represents the time required to collect a 3D tomographic dataset. (b) Shows the force versus displacement plot for the same sample. (c) Shows the 3D tomography surface rendering for the same sample under no load (1) and after transition to plastic deformation (2). 
a)
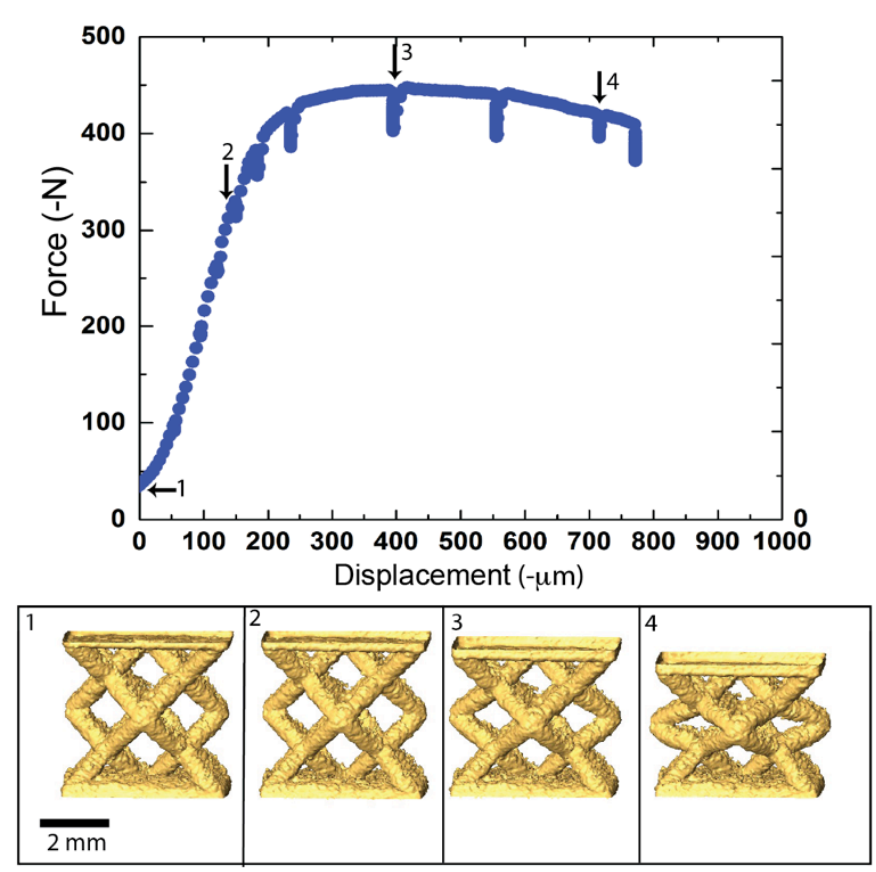

b)
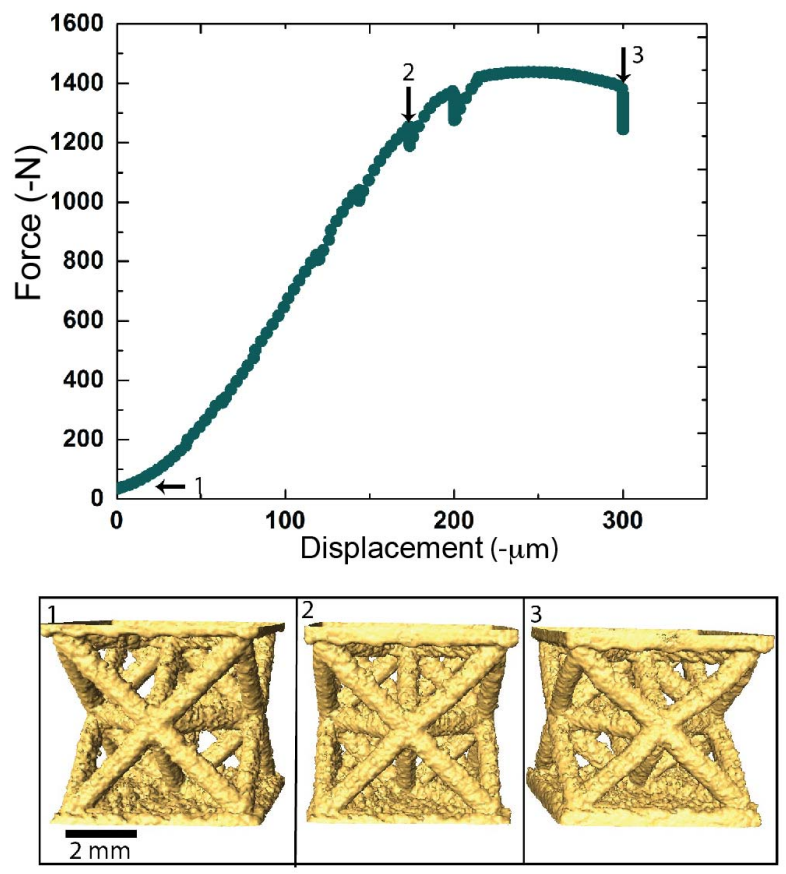

Figure 4. In situ compression results for $20 \%$ relative density samples. Plots showing force versus displacement curves for the $20 \%$ relative density OT (a) and RD (b). Tomography was acquired at different points during deformation and representative 3D reconstructed images are shown below each plot. The numbers correspond to a specific force and displacement marked on each plot. 
a)

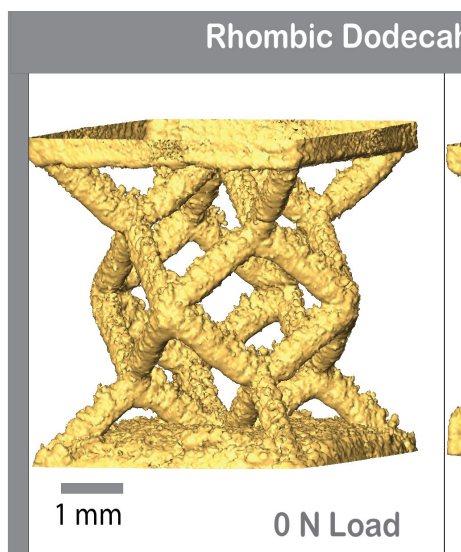

b)

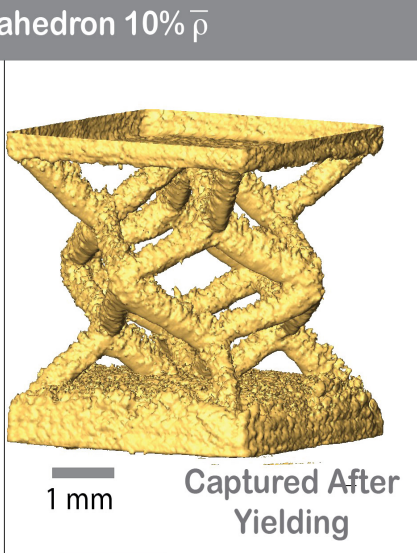

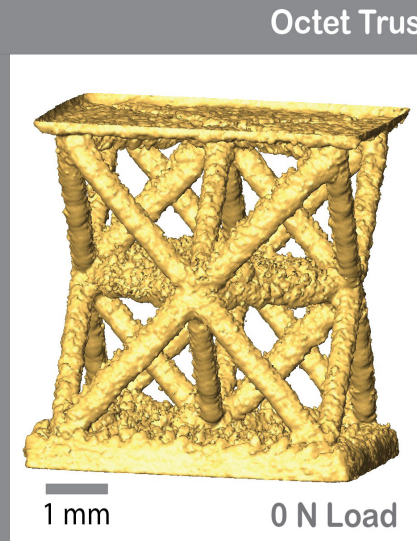
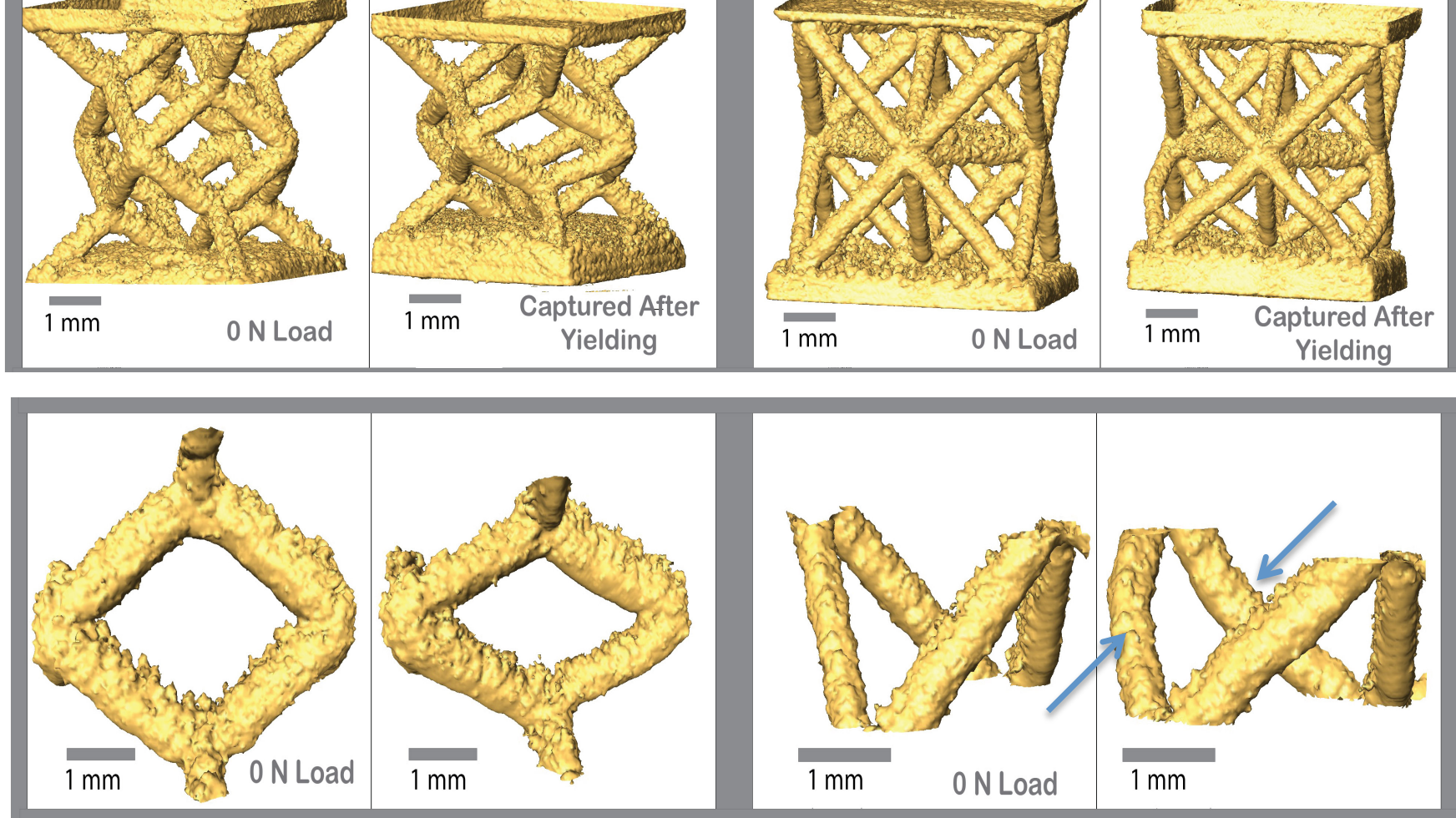

Figure 5. Comparing 3D images of deformed lattice structures. 3D tomographic images showing a RD (a) and an OT (b) before (left) and after deformation (right). The images in (a) shows the crushing or yielding behavior of the RD structure, and the image in (b) show the buckling observed in OT structures with low relative densities (10\%). 


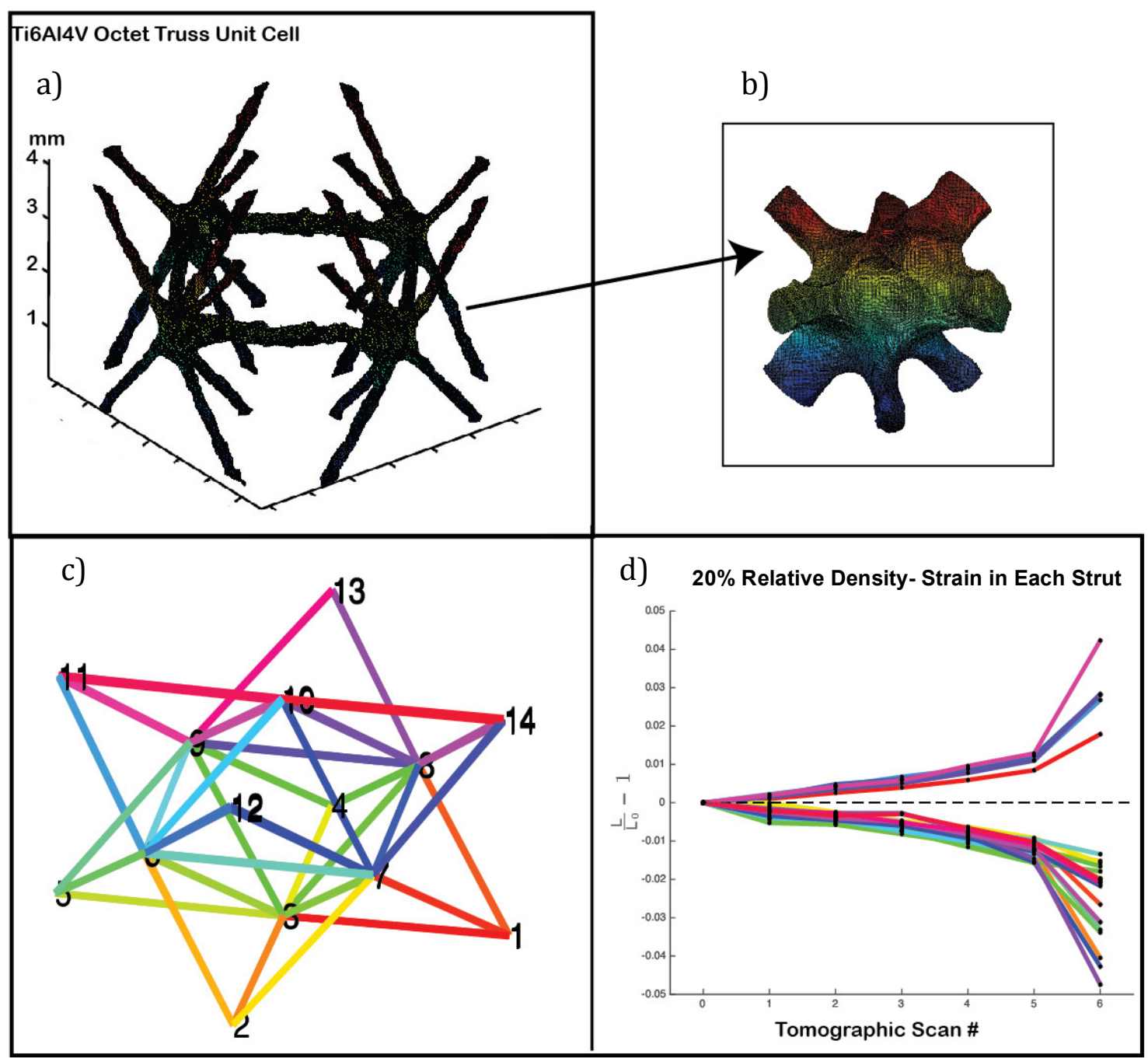

Figure 6. Mapping localized strain in lattice structures. Ball and stick representative of an OT unit cell as measured by tomography: (a) Ligament (or edge) image, (b) isosurface rendering of a single node from an OT lattice at a given scan \# and strain, (c) example of the spatial map connecting nodes, and (d) a graph of localized strain versus tomographic scan \#, where colors represent specific struts. 

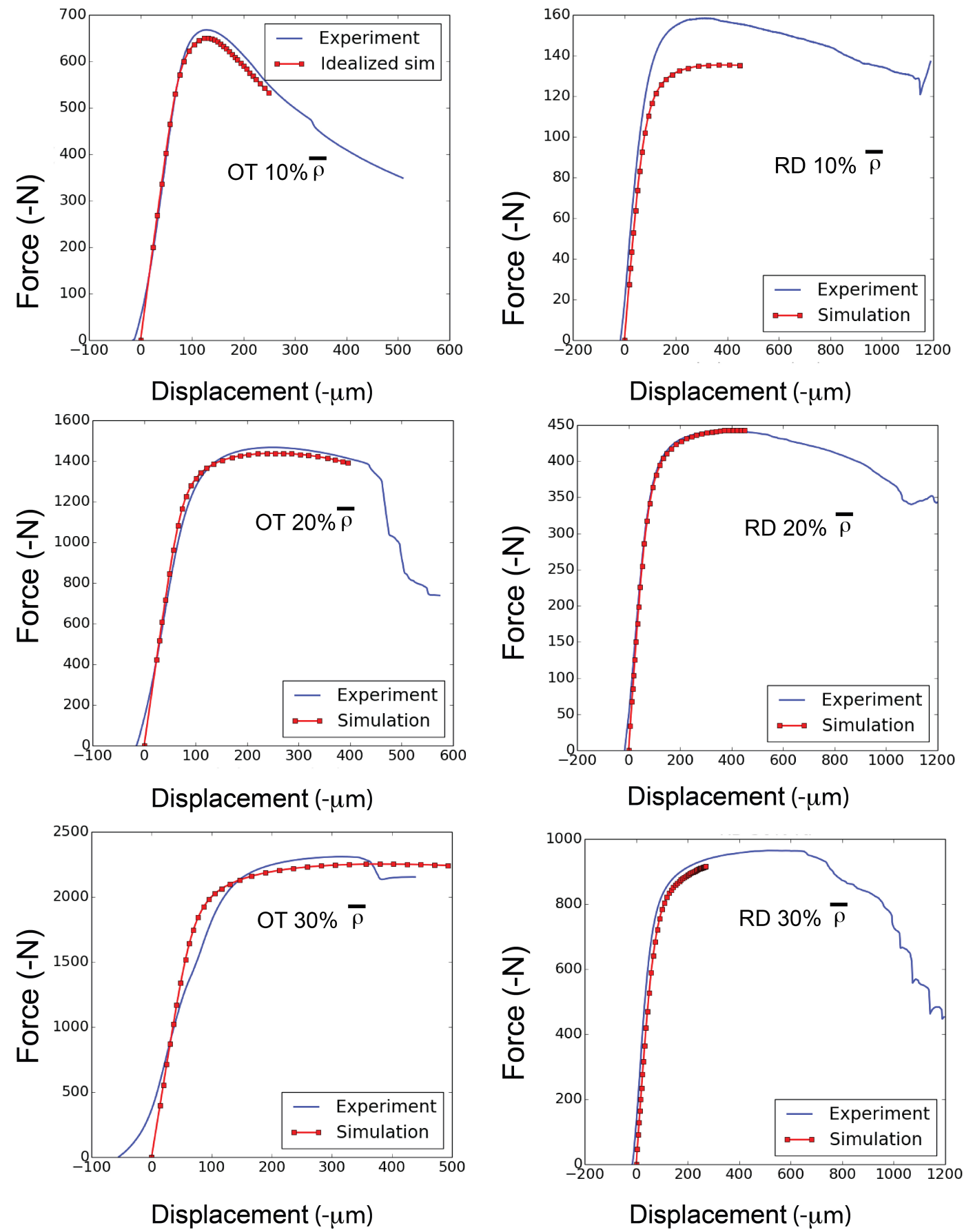

Figure 7. Experimental results versus simulation. Force versus displacement curves showing the comparison between the simulation using a finite element model and the experimental results for the 6 sample groups tested. 
$10 \%$ Octet-Truss Unit Cell
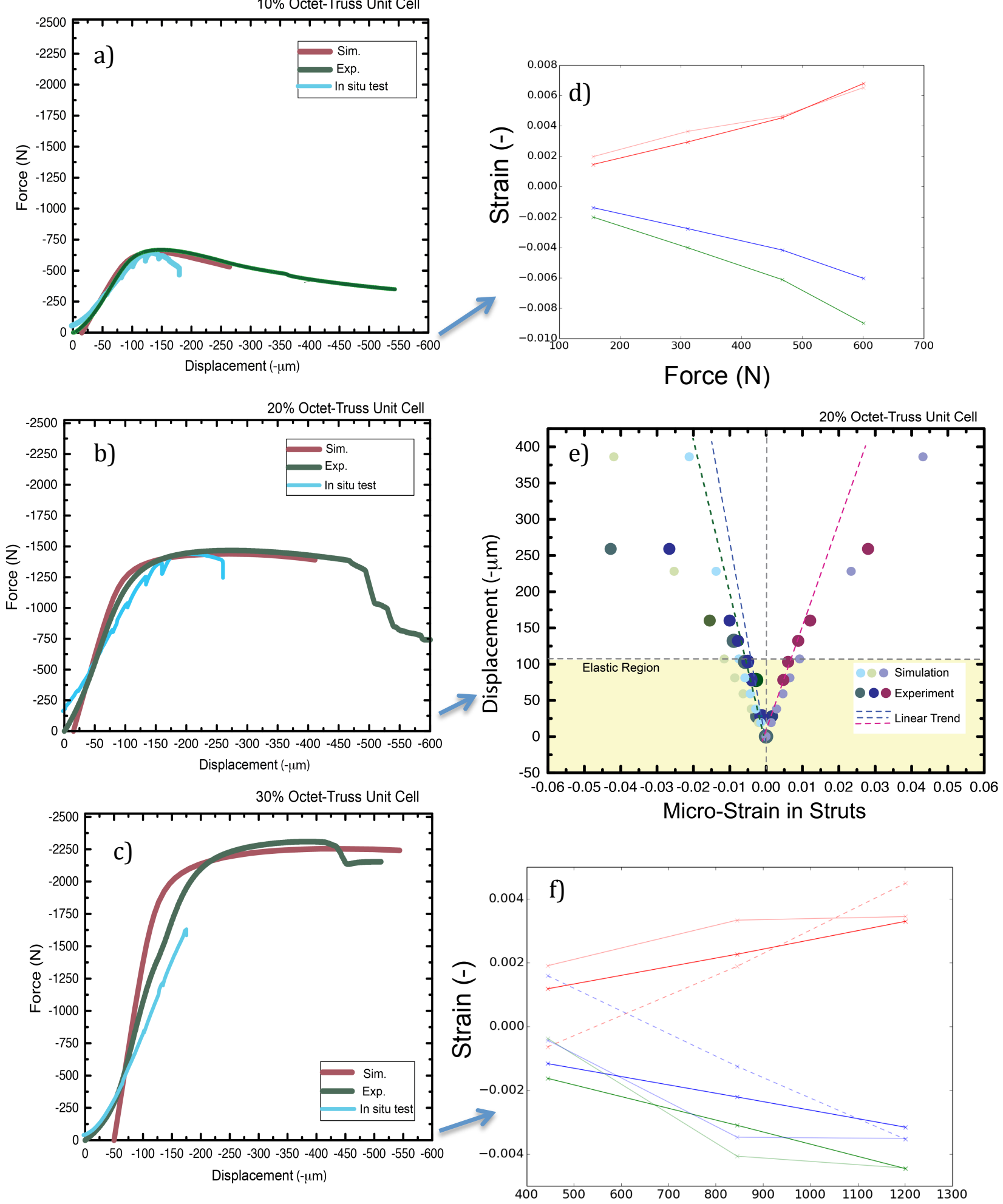

Force $(\mathrm{N})$ 
Figure 8. Experimental results versus simulation for macro- and micro-deformation. Force versus displacement curves for the OT, showing the comparison between both types of experimental compression tests and simulation predictions using the finite element model for samples with a relative density of $10 \%$ (a), 20\% (b) and 30\% (c). The curves in (d) - (f) show the comparison between micro-strain in individual struts determined by experimental results and the finite element model simulation. The tomographic data was used to quantify ligament-average strain for each of the load steps. Here red lines represent tension and green/blue lines represent different levels of compression, the darker lines are from the simulation and the lighter lines are from the experimental results. The dotted lines in 30\% show where the results deviated from the predicted behavior. Plot (e) is shown with different axis (displacement versus micro-strain) to show that the deformation behavior deviates from being linear in the macro-elastic region. 
Octet-truss $30 \%$ relative density
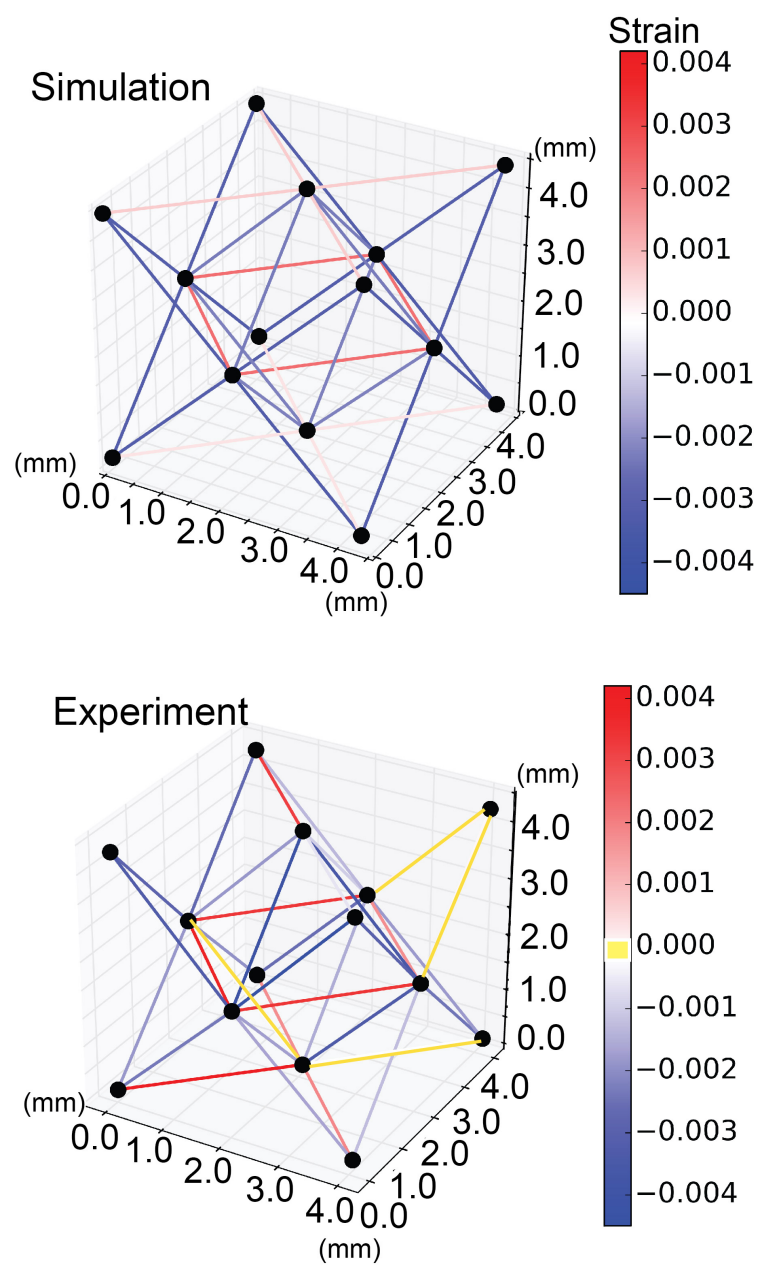

Figure 9. Micro-strain in struts - Simulation versus experiment for the Octet 30\% relative density. The 3D pseudocolor plot compares the strain levels in each strut at a load of 845 N. Red indicates tension while blue indicates compression. There are struts that are taking more strain than predicted due to imperfections in the sample. 


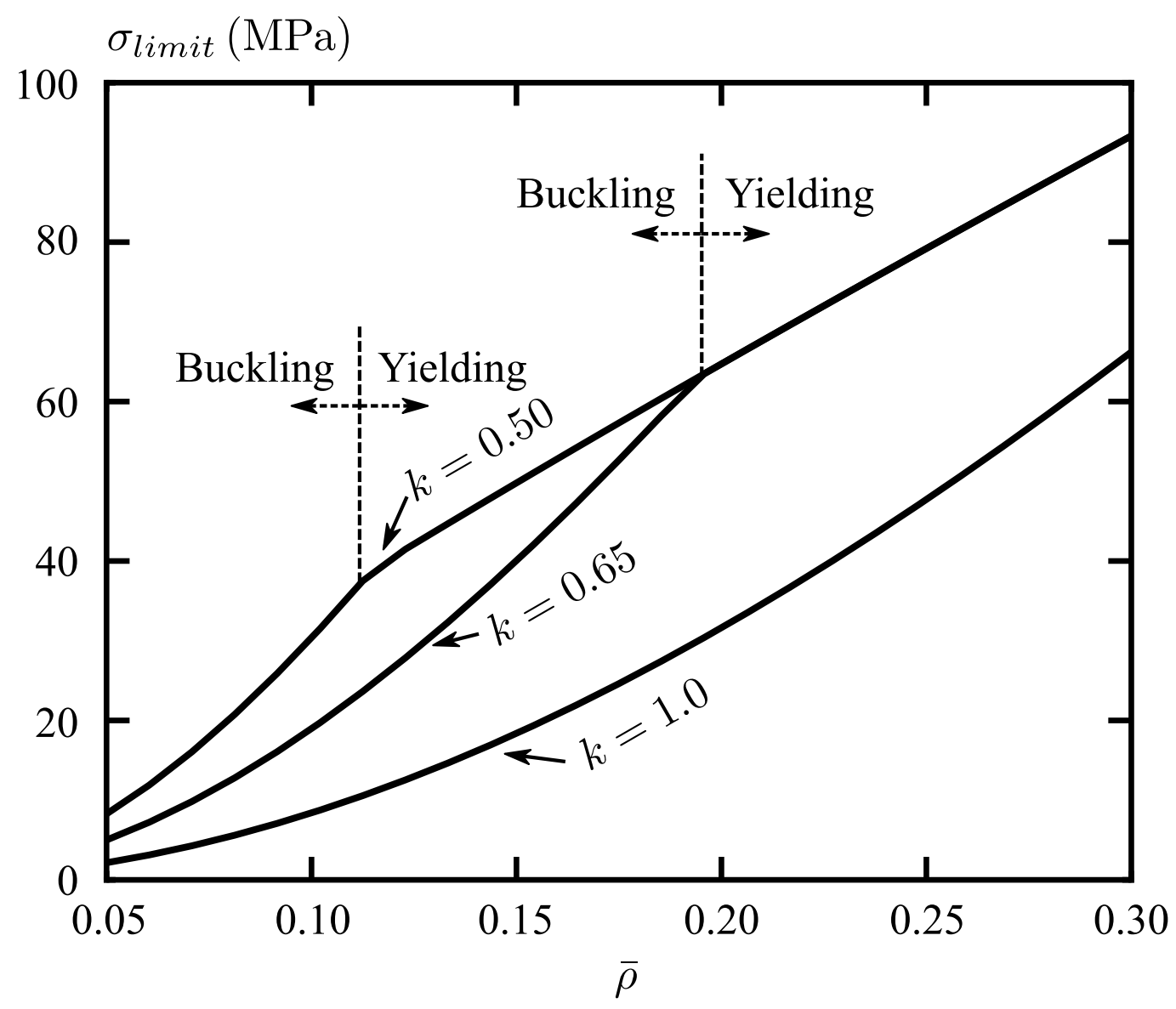

Figure 10. Predicting the transition in failure mode for stretch-dominated lattices. Figure showing the scaling relationship derived from the equivalent beam model. The different strength lines represent different end boundary conditions. The change in slope seen for lines using the boundary conditions $\mathrm{k}=0.5$ and 0.65 show the transition between buckling and yielding at different relative densities. 